\title{
Quasiclassical and Statistical Properties of Fermion Systems
}

\author{
M. Grigorescu and W. E. Baylis \\ Department of Physics, University of Windsor, Windsor, ON, Canada N9B 3P4
}

(Received textdate)

\begin{abstract}
A quasiclassical correspondent for the fermion degrees of freedom is obtained by using a timedependent variational principle with Grassmann coherent states as trial functions. In the real parametrization provided by the canonical coordinates, these states satisfy a closure relationship, and this is used to calculate the partition function at finite temperature. The particular example considered here consists of a many-fermion system in a quantum double-well. Entanglement between the one-particle orbital states leads to deviations from the normal Fermi-Dirac distribution. This deviation is reflected in the dependence of the chemical potential on concentration. In the physical example of two adjacent $\mathrm{CuO}$ planes in a high- $T_{c}$ superconductor, the dependence is suggestive of the pseudogap observed at temperatures $T>T_{c}$.
\end{abstract}




\section{INTRODUCTION}

The classical limit of an elementary quantum dynamical system can show spectacular variation in the nature and number of the degrees of freedom. While the quantum dynamics is mainly stationary, or in transitory regimes given by a linear superposition of an infinite number of harmonic-oscillator modes (the stationary states), the classical dynamics is in general nonstationary and nonlinear, and it involves a relatively small number of degrees of freedom.

The transition from quantum to classical statistics in a many-particle system at finite temperature, resulting from a change in the underlying microscopic dynamics from quantum to classical, is less well explored. The usual approach is based on the Wigner-Kirkwood expansion of the quantum partition function in powers of the Planck constant $h$. However, convergence of this series is assured only when the thermal energy $k_{B} T$ is large with respect to the level spacing [1].

Of course, the classical Maxwell-Boltzmann statistics can be recovered from the quantum Bose-Einstein or Fermi-Dirac statistics in the limit of high temperatures. However, this limit is not exactly a classical limit because the constituent particles remain quantum objects. Moreover for spin- $\frac{1}{2}$ particles, a classical correspondent exists only in the sense of a pseudomechanics, defined in terms of Grassmann variables [2].

Classical degrees of freedom at the quantum level can be introduced with the concepts of a molecular (or nuclear) mean field or of spontaneous symmetry breaking. In such situations, the infinite degeneracy of a metastable ground state is lifted by the emergence of a small number of collective (mean-field) degrees of freedom. At low energy, the collective motion is quantized, but with increasing energy, a transition to classical behavior may occur.

The thermodynamics of quantum many-body systems can be affected not only by the classical behavior of certain degrees of freedom of the constituent particles, but also by macroscopic quantum coherence phenomena. Such phenomena occur, for example, when a quantum system is split by a potential barrier into two entangled components localized in distinct classical regions. This situation appears in the generic case of two quasi-2D (twodimensional) layers of electron gas separated by a thin insulator. Examples of such bilayer structures that are particularly important for applications are provided by the $\mathrm{CuO}$ planes of high- $T_{c}$ superconductors [3] and by the tunnel junction of the recently developed 2D-2D 
transistor [4, 5].

A suitable framework for understanding the quasiclassical behavior of quantum systems is provided by the time-dependent variational principle [6]. This principle leads to quasiclassical dynamics, obtained by constraining the quantum dynamics from the infinite-dimensional Hilbert space to a finite-dimensional manifold of trial functions. The usual mean-field approximations of many-body theory can be recovered in this framework by choosing trial functions that are coherent states of a semisimple Lie group. However, by the choice of the generators, these approximations provide the classical analog for particle-hole or particleparticle operators rather than for individual fermion fields.

A direct quasiclassical correspondent of the Fermi operators can be obtained by using as trial functions the Grassmann coherent states [2, 7]. In Sect. II it will be shown that by a suitable definition of Hermitian conjugation and of the product between conjugate variables, the Grassmann coherent states admit a real parameterization. With respect to this parametrization, the variational principle associates a classical Hamiltonian system for each fermion degree of freedom. The integral of the projector on the Grassmann coherent states, taken over the invariant volume element associated with the canonical variables, provides a resolution of the identity in the many-fermion Hilbert space (see Appendix). This important relationship was obtained previously [8, 9] by direct integration over Grassmann variables. However, the approach based on the Berezin integral [10] may lead to ambiguities, especially in the integration of multiple products involving dual Grassmann variables. As we show, the present definition removes the difficulties encountered in the semiclassical quantization of the phase integrals [11, 12] and leads to the correct Bohr-Wilson-Sommerfeld formula.

The resolution of the identity allows us to reduce the calculus of the trace in many-fermion Hilbert space to an integral over Grassmann coherent states, and this is particularly suited for statistical calculations. At the end of Sect. II, we show that the present approach leads to the standard Fermi-Dirac distribution function.

The effect of quantum entanglement on the particle distribution functions in coupled Fermi systems is discussed in Sect. III. The particular coupling selected here is tunneling through a potential barrier, but this is only a heuristic model. Similar results can be obtained by using any interaction that splits the degenerate levels. It is seen that the level splitting and the difference between the chemical potentials of the two components combine in a single quantity that determines the entangled distribution function. In the physical 
example of the $\mathrm{CuO}$ planes in the high- $T_{c}$ compound $\mathrm{La}_{2-x} \mathrm{Sr}_{x} \mathrm{CuO}_{4}$, we show in Sect. IV that the entanglement brings the normal Fermi-Dirac distribution closer to one of a superconductor. An observable directly related to the distribution function is the shift of the chemical potential as a function of concentration. It is shown that despite the simplicity of the model, the shift calculated for an entangled Fermi-Dirac distribution is in reasonable agreement with available experimental data. A summary of the main results, and the concluding remarks are presented in Sect. V.

\section{GRASSMANN COHERENT STATES AND PARTITION FUNCTIONS}

Consider a model space containing a set of $L$ orthonormal one-particle fermion states $\left\{\psi_{i}, i=1, \ldots, L\right\}$, and let $a_{i}^{\dagger}\left(a_{i}=\left(a_{i}^{\dagger}\right)^{\dagger}\right)$ be the associated fermion creation (annihilation) operators in Fock space with respect to the vacuum state $|0\rangle, a_{i}|0\rangle=0$, such that $\psi_{i}(x)=$ $\left\langle x\left|a_{i}^{\dagger}\right| 0\right\rangle,\left\{a_{i}, a_{j}\right\}=a_{i} a_{j}+a_{j} a_{i}=0$ and $\left\{a_{i}^{\dagger}, a_{j}\right\}=\delta_{i j}$. Coherent fermion states $|\zeta\rangle$ can be defined as eigenstates of the fermion annihilation operator $a_{i}$, with eigenvalues $\zeta_{i}$ [2], 7]

$$
a_{i}|\zeta\rangle=\zeta_{i}|\zeta\rangle
$$

This definition, together with the anticommutivity of the fermion operators, leads to

$$
\left\{a_{i}, a_{j}\right\}|\zeta\rangle= \pm\left\{\zeta_{j}, \zeta_{i}\right\}|\zeta\rangle=0
$$

with the upper $(+)$ sign on the right-hand side if $a_{i}$ and $\zeta_{j}$ commute, $\left[a_{i}, \zeta_{j}\right]=0$, and the lower $(-)$ sign if they anticommute, $\left\{a_{i}, \zeta_{j}\right\}=0$. In both cases $\left\{\zeta_{i}, \zeta_{j}\right\}=0$, which means that the "eigenvalues" $\zeta_{i}, i=1, \ldots, L$ cannot be scalars but should be considered Grassmann variables. Thus, coherent fermion states exist only in a Grassmannian extension of Fock space.

Let $N$ be the number of independent variables among $\zeta_{i}, i=1, \ldots, L$, and let $\left\{w_{\alpha}\right\}$, $\alpha=1, \ldots, N \leq L$, be a basis in the complex linear space $\Gamma_{N}$ of the $\zeta_{i}$. The product of $k \leq N$ distinct basis elements is an element of an $\left(\begin{array}{l}N \\ k\end{array}\right)$-dimensional linear space $\mathcal{G}_{N}^{k}$. For example, $\mathcal{G}_{N}^{0}$ denotes the space of complex scalars and $\mathcal{G}_{N}^{1} \equiv \Gamma_{N}$. The direct sum $\bigoplus_{k=0}^{N} \mathcal{G}_{N}^{k}$ is the $2^{N}$-dimensional linear space of the full Grassmann algebra $\mathcal{G}_{N}$.

To calculate matrix elements of many-body fermion operators between Grassmann coherent states, we must define the Hermitian conjugation operation " $\nmid$ " not only for operators 
but also for Grassmann variables. The operation is introduced here by using the linear space $\Gamma_{N}^{*}$ dual to $\Gamma_{N}$, that is the linear space of complex linear functionals on $\Gamma_{N}$. The basis in $\Gamma_{N}^{*}$ dual to the basis $\left\{w_{\alpha}\right\}$ of $\Gamma_{N}$ comprises functionals $w_{\alpha}^{*}$ defined in terms of unique scalar-valued mappings "." such that $w_{\alpha}^{*} \cdot w_{\beta} \equiv w_{\beta} \cdot w_{\alpha}^{*}=\delta_{\alpha \beta}$ [13]. This relation allows us to introduce the involution operator $*: \Gamma_{N} \rightarrow \Gamma_{N}^{*}$, related to the Hermitian conjugation by $\left(\zeta_{j} a_{i}\right)^{\dagger}=a_{i}^{\dagger} \zeta_{j}^{*}$, such that for any $\zeta_{i}, \zeta_{j} \in \Gamma_{N},\left(\zeta_{j}^{*}\right)^{*}=\zeta_{j},\left(\zeta_{i} \zeta_{j}\right)^{*}=\zeta_{j}^{*} \zeta_{i}^{*}$, and $\left(z \zeta_{j}\right)^{*}=z^{*} \zeta_{j}^{*}$, where $z$ is any complex number and $z^{*}$ is its complex conjugate.

It is often convenient to think of the scalar-valued mappings denoted by "." as products in the direct-sum space $\mathcal{C}_{N}=\Gamma_{N}^{*} \oplus \Gamma_{N}$. Duality then assigns a complex scalar value to any product $\zeta_{j}^{*} \cdot \zeta_{k}=\sum_{\alpha=1}^{N} z_{j}^{\alpha *} z_{k}^{\alpha}$ of the Grassmann variables $\zeta_{j}^{*}=\sum_{\alpha=1}^{N} z_{j}^{\alpha *} w_{\alpha}^{*} \in \Gamma_{N}^{*}$ and $\zeta_{k}=\sum_{\alpha=1}^{N} z_{k}^{\alpha} w_{\alpha} \in \Gamma_{N}$. The space $\mathcal{C}_{N}$ generates a $2^{2 N}$-dimensional Clifford algebra $C \ell_{N, N}$ 13, 14, 15 in which the scalar product of null vectors $\zeta_{j}^{*}$ and $\zeta_{k}$ can be identified with the anticommutator: $\zeta^{*} \cdot \zeta^{\prime}=\frac{1}{2}\left\{\zeta^{*}, \zeta^{\prime}\right\}$ of their associative Clifford product. Physical quantities are evaluated as the scalar part of expressions in $C \ell_{N, N}$, and unless otherwise noted, this restriction is assumed in expressions of expectation values below.

When $L=1$, an unnormalized Grassmann coherent state can be expressed as

$$
|\tilde{\zeta}\rangle=e^{ \pm \zeta_{1} a_{1}^{\dagger}}|0\rangle=\left(1 \pm \zeta_{1} a_{1}^{\dagger}\right)|0\rangle=|0\rangle \pm \zeta_{1}|1\rangle
$$

where $|1\rangle=a_{1}^{\dagger}|0\rangle$. Since the (scalar part of the) overlap

$$
\langle\tilde{\zeta} \mid \tilde{\zeta}\rangle=1+\zeta_{1}^{*} \cdot \zeta_{1}
$$

is a real number, the normalized state (11) is

$$
|\zeta\rangle=\frac{\left(1 \pm \zeta_{1} a_{1}^{\dagger}\right)|0\rangle}{\sqrt{1+\zeta_{1}^{*} \cdot \zeta_{1}}}
$$

It may be verified that since $a_{1} a_{1}^{\dagger}|0\rangle=\left\{a_{1}, a_{1}^{\dagger}\right\}|0\rangle=|0\rangle$ and $\zeta_{1}^{2}=0$,

$$
\begin{aligned}
a_{1}|\zeta\rangle & =\frac{\zeta_{1} a_{1} a_{1}^{\dagger}|0\rangle}{\sqrt{1+\zeta_{1}^{*} \cdot \zeta_{1}}}=\frac{\zeta_{1}|0\rangle}{\sqrt{1+\zeta_{1}^{*} \cdot \zeta_{1}}} \\
& =\frac{\zeta_{1}\left(1 \pm \zeta_{1} a_{1}^{\dagger}\right)|0\rangle}{\sqrt{1+\zeta_{1}^{*} \cdot \zeta_{1}}}=\zeta_{1}|\zeta\rangle .
\end{aligned}
$$

In the $L$-level case it is convenient to assume that $\zeta_{i}$ and $a_{j}^{\dagger}$ anti-commute, and we therefore choose the "-" sign in Eq. (3). Then the normalized Grassmann coherent state has the 
general expression

$$
|\zeta\rangle=\prod_{k=1}^{L} \frac{\left(1-\zeta_{k} a_{k}^{\dagger}\right)}{\sqrt{1+\zeta_{k}^{*} \cdot \zeta_{k}}}|0\rangle=\prod_{k=1}^{L} \frac{\exp \left(-\zeta_{k} a_{k}^{\dagger}\right)}{\sqrt{1+\zeta_{k}^{*} \cdot \zeta_{k}}}|0\rangle .
$$

The expectation value of the particle number operator $a_{k}^{\dagger} a_{k}$ in this state is

$$
n_{k}=\left\langle\zeta\left|a_{k}^{\dagger} a_{k}\right| \zeta\right\rangle=\left\langle\zeta\left|\zeta_{k}^{*} \zeta_{k}\right| \zeta\right\rangle=\frac{\zeta_{k}^{*} \cdot \zeta_{k}}{1+\zeta_{k}^{*} \cdot \zeta_{k}} .
$$

Note that $\zeta_{k}^{*} \zeta_{k}$ is not a pure scalar in $C \ell_{N, N}$ (it also contains a bivector part), and since the coherent state $|\zeta\rangle$ is also not a Grassmann scalar, we do not restrict $\zeta_{k}^{*} \zeta_{k}$, but only its expectation value $\left\langle\zeta\left|\zeta_{k}^{*} \zeta_{k}\right| \zeta\right\rangle$, to its scalar part. When $N=L$, it is possible to choose $\zeta_{k}=z_{k} w_{k}$. In this case, $\zeta_{k}^{*} \cdot \zeta_{k}=z_{k}^{*} z_{k}$ and the absolute value of the complex variable $z_{k}=\rho_{k} e^{i \phi_{k}}$ is related to the particle number by $n_{k}=\rho_{k}^{2} /\left(1+\rho_{k}^{2}\right)$. This relation limits $n_{k}$ to the range from 0 (when $\rho_{k}=0$ ) to 1 (when $\rho_{k} \rightarrow \infty$ ). With such parametrization, Grassmann coherent states become su (2) coherent states (see Appendix). The values of $n_{k}$ are quantized by the anticommutation relation $\left\{a_{i}^{\dagger}, a_{j}\right\}=\delta_{i j}$ or by the old quantum theory (see below).

A quasiclassical approximation of the quantum many-fermion dynamics can be obtained by using the Grassmann coherent state $|\zeta\rangle$ as trial function in the time-dependent variational principle. If $|\zeta\rangle$ depends on time only through the complex scalar variables $z_{k}$, then the best approximation for an exact solution of the time-dependent Schrödinger equation satisfies

$$
\delta_{x} \int_{t_{1}}^{t_{2}}\left\langle\zeta\left|i \hbar \partial_{t}-H\right| \zeta\right\rangle d t=0,
$$

where $H$ is the Hamiltonian of the many-fermion system, $t_{1}, t_{2}$ are fixed times, and $\delta_{x}$ denotes the variation of the integral with respect to the real functions of time $\rho_{k}(t)$ and $\phi_{k}(t), k=1, \ldots, L$, denoted together by $x_{k}, k=1, \ldots, 2 L$.

The equations of motion determined by Eq. (9) have the general form

$$
\sum_{i=1}^{2 L} \dot{x}_{i} \omega_{i j}=\frac{\partial \mathcal{H}}{\partial x_{j}}
$$

where $\mathcal{H}=\langle\zeta|H| \zeta\rangle$, and $\omega_{i j}=-\omega_{j i}=2 \hbar \Im\left(\left\langle\partial_{i} \zeta \mid \partial_{j} \zeta\right\rangle\right)$ are the coefficients of a nondegenerate 2-form (a symplectic structure) on the manifold of Grassmann coherent states. A canonical transformation to the new variables

$$
q_{k}=\sqrt{\frac{2 \hbar}{1+\rho_{k}^{2}}} \sin \phi_{k}, p_{k}=\sqrt{\frac{2 \hbar}{1+\rho_{k}^{2}}} \cos \phi_{k},
$$


defined on the disk of radius $\sqrt{2 \hbar}$ in the $p q$ plane, reduces further the equations of motion to

$$
\dot{q}_{k}=\frac{\partial \mathcal{H}}{\partial p_{k}}, \dot{p}_{k}=-\frac{\partial \mathcal{H}}{\partial q_{k}} .
$$

If the states $\psi_{i}, i=1, \ldots, L$ are one-particle energy eigenstates with eigenvalues $\epsilon_{i}$, then a many-fermion Hamiltonian of the form $H_{f}=\sum_{k=1}^{L} \epsilon_{k} a_{k}^{\dagger} a_{k}$ can be considered as the Hamiltonian of a "Fermi oscillator." Since

$$
q_{k}^{2}+p_{k}^{2}=\frac{2 \hbar}{1+\rho_{k}^{2}}=2 \hbar\left(1-n_{k}\right),
$$

for the Fermi oscillator $\mathcal{H}_{f}=\sum_{k=1}^{L} \epsilon_{k}\left[1-\left(p_{k}^{2}+q_{k}^{2}\right) / 2 \hbar\right]$, and the equations of motion have the solution $\rho_{k}(t)=$ const, $\phi_{k}(t)=-t \epsilon_{k} / \hbar$. This represents a harmonic oscillation of the variables $p_{k}$ and $q_{k}$ at the angular frequency $\epsilon_{k} / \hbar$. Moreover, in this particular case, the Grassmann coherent state $|\zeta\rangle(t)$ is not an approximation but an exact solution of the time-dependent Schrödinger equation,

$$
i \hbar \frac{\partial|\zeta\rangle}{\partial t}=H_{f}|\zeta\rangle
$$

The "classical" variables $p_{k}$ and $q_{k}$, have been introduced without reference to the physical degrees of freedom of the particles (space coordinates + spin). However, it is interesting to note that they behave in all respects as real classical variables. In the $p q$ plane the orbits are circles of radius $\sqrt{2 \hbar\left(1-n_{k}\right)}$. The Bohr-Wilson-Sommerfeld quantization formula selects only the orbits having an area which is an integer multiple of the Planck constant $h$, such that

$$
2 \pi \hbar\left(1-n_{k}\right)=n h, n=0,1, \ldots
$$

This shows that $1-n_{k}$ should be a non-negative integer, and the only possible values of $n_{k}$ are 0 and 1.

The remarkable property of Grassmann coherent states of providing a resolution of the identity operator in many-body Hilbert space (see Appendix)

$$
\mathcal{I}=\frac{1}{\pi^{L}} \int d^{L} \Omega|\zeta\rangle\langle\zeta|
$$

makes them particularly suitable for applications in statistical mechanics. The partition function $Z$ of a quantum $N_{p}$-body system is defined by the trace in Fock space, 
$Z=\operatorname{Tr}\left(e^{-\beta H}\right)=\sum_{[n]}\left\langle[n]\left|e^{-\beta H}\right|[n]\right\rangle$, where $\beta=1 / k_{B} T, H$ is the many-body Hamiltonian operator, and $|[n]\rangle \equiv\left|n_{1} n_{2} \ldots\right\rangle$ represents one of a complete set of states, labeled by the occupation numbers $n_{k}$ of the single-particle levels, and restricted by the condition $\sum_{k} n_{k}=N_{p}$. If these states are eigenstates of $H$ with eigenvalues $E_{[n]}$, then $Z=\sum_{[n]} e^{-\beta E_{[n]}}$. Moreover, if $E_{[n]}=\sum_{k} n_{k} \epsilon_{k}$, then $Z=\sum_{[n]} \prod_{k} e^{-\beta n_{k} \epsilon_{k}}$. The sum $\Sigma_{[n]}$ can be calculated if the condition of having a fixed number of particles is relaxed and the $n_{k}$ are independent [16]. The average number of particles can still be fixed by introducing the chemical potential $\mu$; then $Z=\prod_{k} Z_{k}$ becomes the product of one-level partition functions $Z_{k}=\Sigma_{n_{k}} \exp \left[-\beta n_{k}\left(\epsilon_{k}-\mu\right)\right]$ with $n_{k}=0,1$ for Fermi-Dirac statistics and $n_{k}=0, \ldots, \infty$ for Bose-Einstein.

The statistical significance of the "Fermi oscillator" appears when the corresponding partition function $Z_{f}$ is calculated as an integral over the manifold of Grassmann coherent states. The resolution of the identity presented in the Appendix allows us to write

$$
\begin{aligned}
Z_{f} & =\operatorname{Tr}\left[e^{-\beta\left(H_{f}-\mu N_{T}\right)}\right]=\prod_{k=1}^{L} Z_{k} \\
& =\frac{1}{\pi^{L}} \int d^{L} \Omega\left\langle\zeta\left|e^{-\beta\left(H_{f}-\mu N_{T}\right)}\right| \zeta\right\rangle,
\end{aligned}
$$

where $N_{T}=\sum_{k} a_{k}^{\dagger} a_{k}$ is the total particle number operator and

$$
Z_{k}=\frac{1}{\pi} \int d \Omega_{k}\left\langle\zeta_{k}\left|e^{-\beta a_{k}^{\dagger} a_{k}\left(\epsilon_{k}-\mu\right)}\right| \zeta_{k}\right\rangle
$$

Because $d \Omega_{k}=d \xi_{k} d \phi_{k}=d p_{k} d q_{k} / \hbar$, and for any integer power $n,\left\langle\zeta_{k}\left|\left(a_{k}^{\dagger} a_{k}\right)^{n}\right| \zeta_{k}\right\rangle=$ $\left\langle\zeta_{k}\left|a_{k}^{\dagger} a_{k}\right| \zeta_{k}\right\rangle=n_{k}$ with $n_{k}=1-\left(p_{k}^{2}+q_{k}^{2}\right) / 2 \hbar$, we get

$$
\begin{aligned}
Z_{k} & =\frac{1}{\pi \hbar} \int_{p_{k}^{2}+q_{k}^{2} \leq 2 \hbar} d p_{k} d q_{k}\left\{1+\left[e^{-\beta\left(\epsilon_{k}-\mu\right)}-1\right]\left(1-\frac{p_{k}^{2}+q_{k}^{2}}{2 \hbar}\right)\right\} \\
& =1+e^{-\beta\left(\epsilon_{k}-\mu\right)}
\end{aligned}
$$

recovering the standard result for fermions.

If $\epsilon_{k}-\mu, k=1, \ldots, L$, and $T$ are such that $\left|\epsilon_{k}-\mu\right|<<k_{B} T$, then $e^{-\beta\left(\epsilon_{k}-\mu\right)}-1 \approx$ $-\beta\left(\epsilon_{k}-\mu\right)$. In this case, $Z_{f}$ takes the form of a classical partition function, defined by the phase-space integral of $\exp \left[-\beta\left(\mathcal{H}_{f}-\mu \mathcal{N}\right)\right]$ with respect to the volume element $d^{L} \Omega$, where $\mathcal{N}$ is the expectation value of $N_{T}$. 


\section{DISTRIBUTION FUNCTIONS IN ENTANGLED FERMI SYSTEMS}

The interplay among classical localization, quantum coherence, and temperature on the one-particle distribution function plays a central role in the many-fermion fusion or fission reactions and in the operation of tunnel junctions. Localization occurs when a container filled with Fermi gas is split into two identical, classically distinct components. The opposite process, when two separated, identical Fermi systems merge to form a single large system, represents delocalization. In both situations, the intermediate stages can be modeled by two entangled Fermi systems confined in classically distinct potential wells that are separated by a finite barrier.

The problem of a system of fermions placed in a double-well potential at zero temperature was studied previously in the context of the predicted exchange-driven spontaneous transitions from bilayer to monolayer ground-state configurations [17]. The influence of coupling on the distribution function at finite temperature is studied here by considering a system of fermions of mass $M$ placed in a one-dimensional potential, consisting of two identical rectangular potential wells of width $w$ and depth $U$, separated by a distance $d$. The bounded one-particle energy levels in this case are $E_{k}=x_{k}^{2} U$, where $x=x_{k}$ satisfies

$$
\tan (\tilde{w} x)=\frac{2 x \sqrt{1-x^{2}}}{ \pm e^{-\tilde{d} \sqrt{1-x^{2}}}+2 x^{2}-1}
$$

with $\tilde{w}=w \sqrt{2 M U / \hbar^{2}}$ and $\tilde{d}=d \sqrt{2 M U / \hbar^{2}}$. For the fully merged system $\left(d=0, \tilde{w}_{d}=2 \tilde{w}\right)$ as well as for each single well $\left(d=\infty, \tilde{w}_{d}=\tilde{w}\right)$, the $x_{k}$ are solutions of

$$
\tan \left(\tilde{w}_{d} x\right)=\frac{2 x \sqrt{1-x^{2}}}{2 x^{2}-1}
$$

Let us denote by $|k 1\rangle$ and $|k 2\rangle$ the orbital wave functions localized in wells 1 and 2, respectively, which at $d=\infty$ correspond to the same energy level $\epsilon_{k}^{0}=x_{k}^{2} U$. At large but finite $d$, tunneling lifts the degeneracy. The eigenstates of the coupled system are represented by the entangled symmetric and antisymmetric superpositions $\left|k_{ \pm}\right\rangle=(|k 1\rangle \pm|k 2\rangle) / \sqrt{2}$, separated in energy by the tunnel splitting $\Delta_{k}=\epsilon_{k}^{-}-\epsilon_{k}^{+} \approx 2 V_{c}(k)$, where

$$
V_{c}(k)=\frac{x_{k} U}{\tilde{w}} e^{-\tilde{d} \sqrt{1-x_{k}^{2}}}
$$

is the tunneling matrix element. The Hamiltonian of a many-fermion system distributed over the levels of the entangled wells is $H_{e}=\sum_{k m} h_{k m}$, where $m= \pm 1 / 2$ is the $z$-component 
of the spin, and

$$
\begin{aligned}
h_{k m} & =\epsilon_{k}^{-} a_{k_{-} m}^{\dagger} a_{k_{-} m}+\epsilon_{k}^{+} a_{k_{+} m}^{\dagger} a_{k_{+} m} \\
& =\epsilon_{k}^{0}\left(a_{1 k m}^{\dagger} a_{1 k m}+a_{2 k m}^{\dagger} a_{2 k m}\right)+\frac{\Delta_{k}}{2}\left(a_{1 k m}^{\dagger} a_{2 k m}+a_{2 k m}^{\dagger} a_{1 k m}\right) .
\end{aligned}
$$

In a quantum description of the entangled system, the corresponding partition function is $Z_{e}=\operatorname{Tr}\left(e^{-\beta\left(H_{e}-\mu N_{T}\right)}\right)$. However, as long as the two wells remain classically distinct near equilibrium, we can assume that each one develops its own chemical potential such that $Z_{e}=\operatorname{Tr}\left(e^{-\beta\left(H_{e}-\mu_{1} N_{1}-\mu_{2} N_{2}\right)}\right)$. Following the procedure presented in Sect. II, $Z_{e}$ can be reduced to an integral over Grassmann coherent states,

$$
\begin{aligned}
Z_{e} & =\frac{1}{\pi^{2 L}} \int d^{L} \Omega_{1} d^{L} \Omega_{2}\left\langle\zeta\left|e^{-\beta\left(H_{e}-\mu_{1} N_{1}-\mu_{2} N_{2}\right)}\right| \zeta\right\rangle \\
& =\prod_{k=1}^{L} Z_{k \frac{1}{2}} Z_{k-\frac{1}{2}}
\end{aligned}
$$

where

$$
Z_{k m}=\frac{1}{\pi^{2}} \int d \Omega_{1 k m} d \Omega_{2 k m}\left\langle\zeta_{k m}\left|e^{-\beta\left(h_{k m}-\mu_{1} a_{1 k m}^{\dagger} a_{1 k m}-\mu_{2} a_{2 k m}^{\dagger} a_{2 k m}\right)}\right| \zeta_{k m}\right\rangle
$$

and

$$
\left|\zeta_{k m}\right\rangle=\frac{\exp \left(-\zeta_{1 k m} a_{1 k m}^{\dagger}-\zeta_{2 k m} a_{2 k m}^{\dagger}\right)}{\sqrt{\left(1+\zeta_{1 k m}^{*} \cdot \zeta_{1 k m}\right)\left(1+\zeta_{2 k m}^{*} \cdot \zeta_{2 k m}\right)}}|0\rangle
$$

The operator $\exp \left[-\beta\left(h_{k m}-\mu_{1} a_{1 k m}^{\dagger} a_{1 k m}-\mu_{2} a_{2 k m}^{\dagger} a_{2 k m}\right)\right]$ has the expansion

$$
\begin{aligned}
& 1+\left(e^{\alpha_{k}} \cosh \eta_{k}-1\right)\left(a_{1 k m}^{\dagger} a_{1 k m}+a_{2 k m}^{\dagger} a_{2 k m}\right) \\
& +e^{\alpha_{k}} \sinh \eta_{k}\left[\frac{r}{\eta_{k}}\left(a_{1 k}^{\dagger} a_{1 k}-a_{2 k}^{\dagger} a_{2 k}\right)\right. \\
& \left.+\frac{s_{k}}{\eta_{k}}\left(a_{1 k m}^{\dagger} a_{2 k m}+a_{2 k m}^{\dagger} a_{1 k m}\right)\right] \\
& +\left(e^{2 \alpha_{k}}-2 e^{\alpha_{k}} \cosh \eta_{k}+1\right) a_{1 k m}^{\dagger} a_{1 k m} a_{2 k m}^{\dagger} a_{2 k m},
\end{aligned}
$$

where $\alpha_{k}=-\beta\left(\epsilon_{k}^{0}-\left(\mu_{1}+\mu_{2}\right) / 2\right), \eta_{k}=\sqrt{s_{k}^{2}+r^{2}}, s_{k}=\beta \Delta_{k} / 2$, and $r=\beta\left(\mu_{1}-\mu_{2}\right) / 2$. Denoting by $\langle\langle A\rangle\rangle$ the integral

$$
\langle\langle A\rangle\rangle=\frac{1}{\pi^{2}} \int d \Omega_{1 k m} d \Omega_{2 k m}\left\langle\zeta_{k m}|A| \zeta_{k m}\right\rangle
$$


we get

$$
\begin{gathered}
\langle\langle 1\rangle\rangle=4,\left\langle\left\langle a_{1 k m}^{\dagger} a_{1 k m}\right\rangle\right\rangle=\left\langle\left\langle a_{2 k m}^{\dagger} a_{2 k m}\right\rangle\right\rangle=2, \\
\left\langle\left\langle a_{1 k m}^{\dagger} a_{1 k m} a_{2 k m}^{\dagger} a_{2 k m}\right\rangle\right\rangle=1
\end{gathered}
$$

such that $Z_{k m}$ has the expression

$$
Z_{k m}=e^{2 \alpha_{k}}+2 e^{\alpha_{k}} \cosh \eta_{k}+1
$$

The quantity that contains the effect of entanglement on the distribution function is $\eta_{k}=$ $\sqrt{s_{k}^{2}+r^{2}}$. If $\left|s_{k}\right|<<|r|$, then $Z_{k m}$ becomes the product $Z_{k 1 m} Z_{k 2 m}$ of the Fermi distributions for the orbital levels $|k 1\rangle$ and $|k 2\rangle$,

$$
\begin{aligned}
& Z_{k 1 m}=1+e^{-\beta\left(\epsilon_{k}^{0}-\mu_{1}\right)} \\
& Z_{k 2 m}=1+e^{-\beta\left(\epsilon_{k}^{0}-\mu_{2}\right)} .
\end{aligned}
$$

When $|r|<<\left|s_{k}\right|$, then $Z_{k m}$ is the product $Z_{k_{-} m} Z_{k_{+} m}$ of the distributions

$$
\begin{aligned}
& Z_{k_{-} m}=1+e^{-\beta\left(\epsilon_{k}^{-}-\mu\right)} \\
& Z_{k_{+} m}=1+e^{-\beta\left(\epsilon_{k}^{+}-\mu\right)}
\end{aligned}
$$

and $\mu=\left(\mu_{1}+\mu_{2}\right) / 2$ is the Fermi level of the fully merged system.

For the electron layers in the 2D-2D tunneling transistor, the difference between the chemical potentials is related to the applied voltage $V, \mu_{1}-\mu_{2}=e V$ [5]. In the case of two adjacent $\mathrm{CuO}$ layers of a high- $T_{c}$ compound, a small difference may appear due to the slight asymmetry observed in the placement of the oxygen acceptors between the planes [18].

\section{ENTANGLED FERMI DISTRIBUTIONS IN HIGH- $T_{c}$ SUPERCONDUC- TORS}

In high- $T_{c}$ materials the charge carriers are confined to the $\mathrm{CuO}$ planes, but the interlayer coupling is important in both the normal and superconducting states. At intermediate temperatures and low doping, the interlayer coupling is dominated by the magnetic interaction of the electron spins, producing antiferromagnetic ordering. The charge transport between 
the layers, practically absent in this state, is assumed to have both a coherent, momentumconserving component and an incoherent part due to impurity-assisted hopping [19]. With increasing doping, at lower temperature the coupling takes the form of coherent Josephson pair tunneling, supposedly one of the basic phenomena responsible for the superconductivity at high temperatures. In this perspective, the observed decrease of transition temperature $T_{c}$ with an increase of the number of $\mathrm{CuO}$ planes seems to be explained by the loss of the phase coherence [20].

Layered copper oxide compounds are peculiar not only in their high $T_{c}$ values compared to conventional superconductors, but also in various anomalous properties observed in their normal state. Anomalies showing a common trend with respect to the temperature and to concentration of charge carriers are confirmed by a wide variety of experimental techniques [21. Many anomalous features can be interpreted in terms of a "normal-state gap" or pseudogap [9, 21, 22] of unknown origin that turns into the BCS gap when the material becomes superconducting.

The results presented in Sect. III indicate that entanglement between adjacent layers can produce significant deviations from the standard Fermi-Dirac distribution that may contribute to the observed anomalies. The magnitude of such effects is evaluated here within a schematic model in which the energy of the electrons in a $\mathrm{CuO}$ plane without coupling has the simple form

$$
\epsilon_{k}^{0} \equiv \epsilon_{\kappa}^{0}=\epsilon_{p}+\epsilon_{n}^{c}
$$

where $\kappa \equiv\left(\epsilon_{p}, n\right)$ denotes the couple of a continuous index $\epsilon_{p}$ and a discrete index $n$. These indices correspond to the terms $\epsilon_{p}$ and $\epsilon^{c}$ of the total energy due to the motion of the electrons along directions parallel and normal to the planes, respectively. The in-plane energy term is approximated by a single band, $\epsilon_{p} \in\left[\epsilon_{\min }, \epsilon_{\max }\right]$, with $-\epsilon_{\min }=\epsilon_{\max }=1 \mathrm{eV}$, and a Fermi level $\epsilon_{F}=-0.45 \mathrm{eV}$ 23. Confinement is modeled by a one-dimensional potential well placed normal to the planes. As suggested by the expression for the hole binding potential in oxygenated $\mathrm{La}_{2} \mathrm{CuO}_{4+y}$, [18], the potential well is about $35 \mathrm{meV}$ deep and has a radius $a=8 \AA$. Thus, $\epsilon_{k}^{c}$ is calculated by using the square-well estimate $\epsilon_{n}^{c}=-\left(1-x_{n}^{2}\right) U$, $x_{n} \approx n \pi / \tilde{w}<1$, where $U=35 \mathrm{meV}$, and $w=2 a=16 \AA$. The interlayer coupling matrix element $t_{c}$ depends on doping, and in $\mathrm{La}_{2-x} \mathrm{Sr}_{x} \mathrm{CuO}_{4}, t_{c} \sim 0.66 x^{2.974} \mathrm{eV}$ 24]. This shows that $t_{c}$ depends on the hole concentration $\delta \approx x$ and on the band filling $f=1-\delta$. 
The anomalous behavior attributed to the formation of a pseudogap occurs in general in underdoped compounds. In $\mathrm{La}_{2-x} \mathrm{Sr}_{x} \mathrm{CuO}_{4}, T_{c}$ attains a maximum of $\sim 40 \mathrm{~K}$ at $x_{\text {opt }} \sim 0.15$. Measurements of the specific heat coefficient [25] in this compound indicate the existence of a pseudogap for $x<0.22$. In the range $0.1<x<0.22$, the pseudogap has an almost linear decrease with $x$, from $\sim 200 \mathrm{meV}$ to 0 [26]. At $x \sim 0.1$, when the pseudogap is large, the interlayer coupling strength is $t_{c} \sim 1 \mathrm{meV}$. This value is large compared to the one obtained from Eq. (22), indicating that it may include contributions due to other interactions, stronger than tunneling coupling. In the present calculations, the energy splitting $\Delta_{\kappa} \equiv \Delta_{n}=2 V_{c}(n)$ was calculated by taking $V_{c}(n)=0.001+1.5 \sqrt{x_{n}} \mathrm{eV}$. This includes the realistic term $t_{c}=1 \mathrm{meV}$, and a term weakly increasing with the excitation energy along the normal to the plane.

The number of particles $N_{1}$ in the CuO plane 1 is related to the partition function $Z_{\kappa m}$ of Eq. (30) by

$$
N_{1}=\frac{\partial \ln Z}{\partial \beta \mu_{1}}=\sum_{m} \sum_{\kappa} \frac{\partial \ln Z_{\kappa m}}{\partial \beta \mu_{1}} .
$$

Here $\sum_{\kappa}$ is calculated as an integral over the planar band and a sum over the bounded energy levels for motion normal to the plane, such that

$$
\sum_{\kappa} \equiv \sum_{n} N_{p} S \int d \epsilon_{p}
$$

where $N_{p}=m^{*} /\left(2 \pi \hbar^{2}\right)$ is the planar density of states, $m^{*}$ is the effective band mass [28], and $S$ is the plane surface. Thus, the planar density $D_{p}=N_{1} / S$ of charge carriers takes the form

$$
D_{p}=\int_{\epsilon_{\min }}^{\epsilon_{\max }} d \epsilon\langle n\rangle_{(\epsilon)}
$$

where

$$
\langle n\rangle_{(\epsilon)}=2 N_{p} \sum_{n} \frac{e^{\alpha_{\epsilon n}}+\cosh \eta_{n}+r / \eta_{n} \sinh \eta_{n}}{e^{\alpha_{\epsilon n}}+2 \cosh \eta_{n}+e^{-\alpha_{\epsilon n}}},
$$

$\alpha_{\epsilon n}=-\beta\left(\epsilon+\epsilon_{n}^{c}\right)+\beta\left(\mu_{1}+\mu_{2}\right) / 2, \eta_{n}=\sqrt{s_{n}^{2}+r^{2}}, s_{n}=\beta \Delta_{n} / 2$, and $r=\beta\left(\mu_{1}-\mu_{2}\right) / 2$. The density $\langle n\rangle_{(\epsilon)}$ is shown as a function of $\epsilon$ at $T=120 \mathrm{~K}$ and $r=0$ in Fig. 1 (c). For comparison, on the same plot we display (a) the standard Fermi-Dirac distribution obtained in the absence of interlayer coupling $\left(V_{c}=0, s_{n}=0\right)$, and (b) the distribution

$$
\langle n\rangle_{(\epsilon)}^{s c}=N_{p} \sum_{n}\left[1-\frac{\epsilon_{\kappa}^{0}-\epsilon_{F}}{E_{\kappa}}+\frac{2\left(\epsilon_{\kappa}^{0}-\epsilon_{F}\right)}{E_{\kappa}\left(e^{\beta E_{\kappa}}+1\right)}\right]
$$


of a superconducting system at finite temperature [27]. In this expression, the quasiparticle energy $E_{\kappa}=\sqrt{\left(\epsilon_{\kappa}^{0}-\epsilon_{F}\right)^{2}+\Delta_{s c}^{2}}$ was calculated by using a fictitious superconducting gap $\Delta_{s c}=0.6 \mathrm{eV}$.

The comparison between these functions shows that although different, as far as deviations from the normal Fermi-Dirac distribution are concerned, (b) and (c) may account for similar effects. An observable related directly to the particle distribution is represented by the shift $\Delta \mu$ of the chemical potential as a function of concentration. This shift was subject to a detailed experimental investigation, observing that in $\mathrm{La}_{2-x} \mathrm{Sr}_{x} \mathrm{CuO}_{4}$, an anomaly occurs at low doping levels [28].

The comparison of the experimental data with the shift determined by the distribution functions (a),(b) and (c) of Fig. 1 is presented in Fig. 2. The reference Fermi level was fixed at $\epsilon_{F}=-0.45 \mathrm{eV}$, and the hole concentration $\delta=1-f$ was calculated by assuming that $f=\left.D_{p}\right|_{\mu_{1}=\mu_{2}=\epsilon_{F}+\delta \mu} /\left.D_{p}\right|_{\mu_{1}=\mu_{2}=\epsilon_{F}}$. The Fermi-Dirac distribution produces a linear decrease (Fig. 2-a), while $\langle n\rangle_{(\epsilon)}^{s c}$ (Fig. 2-b), and $\langle n\rangle_{(\epsilon)}$ (Fig. 2-c), both lead to a dependence $\Delta \mu \sim \delta^{2}$. The experimental values can be fitted by a quadratic polynomial (Fig. 2-d).

The suppression of the chemical potential shift for small $\delta$ compared to its value at overdoping is taken as an indication for the opening of the pseudogap [28]. The present calculations indicate that the shift produced by an entangled Fermi-Dirac distribution can be very well simulated by introducing a fictitious gap parameter. The entangled distribution leads also to a quadratic dependence of $\Delta \mu$ on $\delta$, as seen in experiment, in contrast to the standard Fermi-Dirac distribution. However, although overall the results are in a reasonable agreement with experimental data, the simple model considered here cannot explain the complete suppression of the chemical potential shift for small $\delta$.

\section{SUMMARY AND CONCLUSIONS}

The effective number of microscopic degrees of freedom in a many-particle system may change when classical and quantum aspects coexist, and such variations are reflected in the expression of thermodynamic and statistical functions. For a classical particle, there are few translational and rotational degrees of freedom. The classical motion can, in principle, be described in terms of an infinite number of fictitious harmonic oscillators in a Fourier transform, but these oscillators have no statistical relevance. The same holds to a certain 
degree in the case of a quantum object. The energy eigenstates are normalized, and have an oscillatory time evolution. However, as shown by the quasiclassical picture of the old quantum mechanics, these eigenstate oscillators provide only a partition of the low-dimensional classical phase space into cells of the size of the Planck constant $h$ rather than representing new physical degrees of freedom. However, new degrees of freedom are represented by the occupation numbers of these cells.

The quasiclassical dynamics of the occupation numbers in a system of fermions at finite temperature was derived here using Grassmann coherent states. We have shown that these states admit a real parametrization in which they represent $s u(2)$ coherent states (Appendix). With respect to this parametrization the expectation value of the occupation number obeys Hamiltonian dynamics (Sect. II), and the Grassmann coherent states satisfy a closure relationship (Appendix). This relationship allows us to recover the standard FermiDirac distribution function (Sect. II) and to calculate the particle density in entangled Fermi systems at finite temperature (Sect. III).

It is interesting to note that within the thermo-field dynamics formalism [29], the trace is calculated as a "thermal vacuum" expectation value. This "temperature-dependent vacuum" is defined in terms of the composite system consisting of the original physical system and an identical but fictitious quantum dynamical system. When the fermion operators of the fictitious system are replaced by Grassmann variables, the "thermal vacuum" takes the form of a Grassmann coherent state.

The entangled Fermi-Dirac distribution can be relevant in the description of coupled 2D-electron systems. As an example, in Sect. IV we have considered the case of two adjacent $\mathrm{CuO}$ planes in the high- $\mathrm{T}_{c}$ superconductor $\mathrm{La}_{2-x} \mathrm{Sr}_{x} \mathrm{CuO}_{4}$. We have shown that entanglement can produce significant deviations from the standard Fermi-Dirac distribution in the normal state. It also leads to a shift of the chemical potential with the concentration that is very close to one of a superconducting distribution at finite temperature. The results are in reasonable agreement with experimental data, indicating that a more detailed study may be important in understanding the pseudogap-related phenomena. 


\section{Appendix}

The Grassmann coherent states of Eq. (77) can also be expressed by the action of a unitary operator on the vacuum as

$$
|\tau\rangle=\exp \left[\sum_{k=1}^{L}\left(\tau_{k} a_{k}^{\dagger}-a_{k} \tau_{k}^{*}\right)\right]|0\rangle .
$$

The relationship between the Grassmann variables $\tau_{k}$ and $\zeta_{k}$ can be obtained by observing that for each $k$, the operators

$$
S_{+}=\frac{\tau_{k}}{\left|\tau_{k}\right|} a_{k}^{\dagger}, S_{-}=a_{k} \frac{\tau_{k}^{*}}{\left|\tau_{k}\right|}, S_{0}=a_{k}^{\dagger} a_{k}-\frac{1}{2},
$$

with $\left|\tau_{k}\right|=\sqrt{\tau_{k}^{*} \cdot \tau_{k}}$ generate an $s u(2)$ algebra, and that

$$
\begin{aligned}
\exp \left(\tau_{k} a_{k}^{\dagger}-a_{k} \tau_{k}^{*}\right)|0\rangle & =\exp \left[\left|\tau_{k}\right|\left(S_{+}-S_{-}\right)\right]|0\rangle \\
& =\cos \left|\tau_{k}\right| \exp \left(\frac{\tan \left|\tau_{k}\right|}{\left|\tau_{k}\right|} \tau_{k} a_{k}^{\dagger}\right)|0\rangle
\end{aligned}
$$

Thus, the expression (39) has the form of the coherent state (7) with $\zeta_{k}=\tau_{k}\left|\tau_{k}\right|^{-1} \tan \left|\tau_{k}\right|$.

With respect to the real parametrization introduced in Sect. II, the Grassmann coherent states provide a resolution of the identity operator $\mathcal{I}$ of the many-particle Hilbert space such that $\mathcal{I}=c_{P} \mathcal{P}$ with

$$
\mathcal{P}=\int d \Omega|\zeta\rangle\langle\zeta|
$$

where $c_{P}$ is the normalization constant and $d \Omega$ is a volume element on the manifold of Grassmann coherent states.

If the model space contains only one fermion state $(L=1)$, the Fock space consists of the vacuum $|0\rangle$ and a one-particle state $|1\rangle=a_{1}^{\dagger}|0\rangle$, and $|\zeta\rangle=\sqrt{\xi_{1}}\left(|0\rangle-\zeta_{1}|1\rangle\right)=$ $\sqrt{\xi_{1}}\left(|0\rangle+|1\rangle \zeta_{1}\right)$ with $\zeta_{1}=z_{1} w_{1}, z_{1}=\rho_{1} e^{i \phi_{1}}$, and $\xi_{1}=1 /\left(1+\rho_{1}^{2}\right)$. In this case

$$
\left\langle n_{1} \mid \zeta\right\rangle=\sqrt{\xi_{1}} \zeta_{1}^{n_{1}}
$$

where $n_{1}=0,1$. The variables $\xi_{1}, \phi_{1}$ are related to $p_{1}$ and $q_{1}$ defined in Eq. (11) by a canonical transformation, and if the volume element is chosen as $d \Omega=d p_{1} d q_{1} / \hbar=d \xi_{1} d \phi_{1}$, then the off-diagonal terms are eliminated by the integration over $\phi_{1}$, leaving

$$
\langle i|\mathcal{P}| k\rangle=2 \pi \delta_{i k} \int d \xi_{1} \xi_{1}\left(\delta_{i 0}+\rho_{1}^{2} w_{1} w_{1}^{*} \delta_{i 1}\right)
$$


Because $\rho_{1}^{2}=\xi_{1}^{-1}-1$ and

$$
\int_{0}^{1} d \xi_{1} \xi_{1}=\int_{0}^{1} d \xi_{1} \xi_{1}\left(\frac{1}{\xi_{1}}-1\right)=\frac{1}{2}
$$

then $\langle i|\mathcal{P}| k\rangle=\pi \delta_{i k}$, which shows that $c_{P}=1 / \pi$.

Similarly, it can be shown that in general, the overlap between the Grassmann coherent state of Eq. (可) and a many-body state $|[n]\rangle \equiv\left|n_{1} n_{2} \ldots n_{L}\right\rangle=\left(a_{1}^{\dagger}\right)^{n_{1}}\left(a_{2}^{\dagger}\right)^{n_{2}} \ldots\left(a_{L}^{\dagger}\right)^{n_{L}}|0\rangle$, $n_{1}, n_{2}, \ldots n_{L}=0,1$, is, up to a \pm 1 factor,

$$
\left\langle n_{1} n_{2} \ldots n_{L} \mid \zeta\right\rangle=\prod_{k=1}^{L} \sqrt{\xi_{k}} \zeta_{k}^{n_{k}} .
$$

By choosing $d^{L} \Omega=\prod_{k=1}^{L} d \xi_{k} d \phi_{k}$, we get

$$
\left\langle n_{1} n_{2} \ldots n_{L}\left|\int d^{L} \Omega\right| \zeta\right\rangle\left\langle\zeta \mid m_{1} m_{2} \ldots m_{l}\right\rangle=\pi^{L} \delta_{[n][m]},
$$

which proves the decomposition of the identity

$$
\mathcal{I}=\frac{1}{\pi^{L}} \int d^{L} \Omega|\zeta\rangle\langle\zeta|
$$

Using this formula we can express the trace of a many-body operator $A$ as an integral over Grassmann coherent states. Thus,

$$
\begin{aligned}
\operatorname{Tr}(A) & =\operatorname{Tr}(A \mathcal{I})=\Sigma_{[n]}\langle[n]|A \mathcal{I}|[n]\rangle \\
& =\frac{1}{\pi^{L}} \int d^{L} \Omega \Sigma_{[n]}\langle[n]|A| \zeta\rangle\langle\zeta \mid[n]\rangle .
\end{aligned}
$$

However, because the $|[n]\rangle$ constitute a complete set of many-particle configurations in the selected subspace, $\sum_{[n]}\langle\zeta \mid[n]\rangle\langle[n]|=\langle\zeta|$, and

$$
\operatorname{Tr}(A)=\frac{1}{\pi^{L}} \int d^{L} \Omega\langle\zeta|A| \zeta\rangle .
$$

\section{Acknowledgments}

Support of this research by the Natural Sciences and Engineering Research Council of Canada is gratefully acknowledged.

[1] J. G. Kirkwood, Phys. Rev. 44, 31 (1933). 
[2] J. L. Martin, Proc. Roy. Soc. (London) A251, 536, 543 (1959).

[3] J. G. Bednorz and K. A. Müller, Z. Phys. B64, 189 (1986).

[4] J. A. Simmons, M. A. Blount, J. S. Moon, S. K. Lyo, W. E. Baca, J. R. Wendt, J. L. Reno and M. J. Hafich, J. Appl. Phys. 845626 (1998).

[5] S. K. Lyo, Phys. Rev. B 618316 (2000).

[6] P. Kramer and M. Saraceno, Geometry of the Time-Dependent Variational Principle in Quantum Mechanics, Lecture Notes in Physics, vol. 140, Springer, New York, 1981.

[7] J. Schwinger, Phys. Rev. 92, 1283 (1953).

[8] G. Junker and J. R. Klauder, quant-ph/9708027 - Eur. Phys. J. C4, 173 (1998).

[9] M. Imada, A. Fujimori and Y. Tokura, Rev. Mod. Phys. 70, 1039 (1998).

[10] F. A. Berezin, The Method of Second Quantization, Academic Press, New York, (1966).

[11] R. Casalbuoni, Il Nuovo Cimento 33A, 115, 389 (1976).

[12] S. Abe and S. Naka, Prog. Theor. Phys. 72, 881 (1984).

[13] C. Doran, D. Hestens, F. Sommen and N. Van Acker, J. Math. Phys. 34, 3642 (1993).

[14] W. E. Baylis, Electrodynamics - A Modern Geometric Approach, Birkhäuser, Boston, (1999), p. 8.

[15] P. Lounesto, Clifford Algebras and Spinors, 2e, Cambridge University Press, Cambridge, UK, (2001).

[16] A. Sommerfeld, Thermodynamik und Statistik, 3 Aufl., Akad. Verl. Ges., Leipzig, (1965).

[17] L. Zheng, M. W. Ortalano, and S. Das Sarma, Phys. Rev. B55, 4506 (1997).

[18] M. A. Kastner, R. J. Birgeneau, G. Shirane and Y. Endoh, Rev. Mod. Phys. 70, 897 (1998).

[19] W. Kim and J. P. Carbotte, cond-mat/0010324

[20] E. Pavarini, I. Dasgupta, T. Saha-Dasgupta, O. Jepsen and O. K. Andersen, condmat/0012051

[21] T. Timusk and B. W. Statt, Rep. Prog. Phys. 62, 61 (1999).

[22] P. W. Anderson, cond-mat/0108522

[23] S. Chakravarty, A. Sudbo, P. W. Anderson and S. Strong, Science 261, 337 (1993).

[24] Y. Zha, S. L. Cooper and D. Pines, Phys. Rev. B 53, 8253 (1996).

[25] J. W. Loram, K. A. Mirza, J. R. Cooper and J. L. Tallon, Physica C 282, 1405 (1997).

[26] A. Fujimori, A. Ino, T. Yoshida, T. Mizokawa, Z.-X. Shen, C. Kim, T. Kakeshita, H. Eisaki and S. Uchida, cond-mat/0011293 
[27] J. M. Blatt, Theory of Superconductivity, Academic Press, New York, (1964), p. 237.

[28] A. Ino, T. Mizokawa, A. Fujimori, K. Tamasaku, H. Eisaki, S. Uchida, T. Kimura T. Sasagawa, and K. Kishio, Phys. Rev. Lett. 79, 2101 (1997).

[29] Y. Takahashi and H. Umezawa, Coll. Phen. 2, 55 (1975). 


\section{Figure captions}

Fig. 1. Planar electron density as a function of energy. (a) normal Fermi-Dirac distribution; (b) superconducting at finite temperature; (c) entangled Fermi-Dirac.

Fig. 2. Chemical potential shift as a function of hole concentration. (*) experimental values; (a) normal Fermi-Dirac distribution; (b) superconducting at finite temperature; (c) entangled Fermi-Dirac; (d) quadratic polynomial fit of the experimenal data. 
Fig. 1

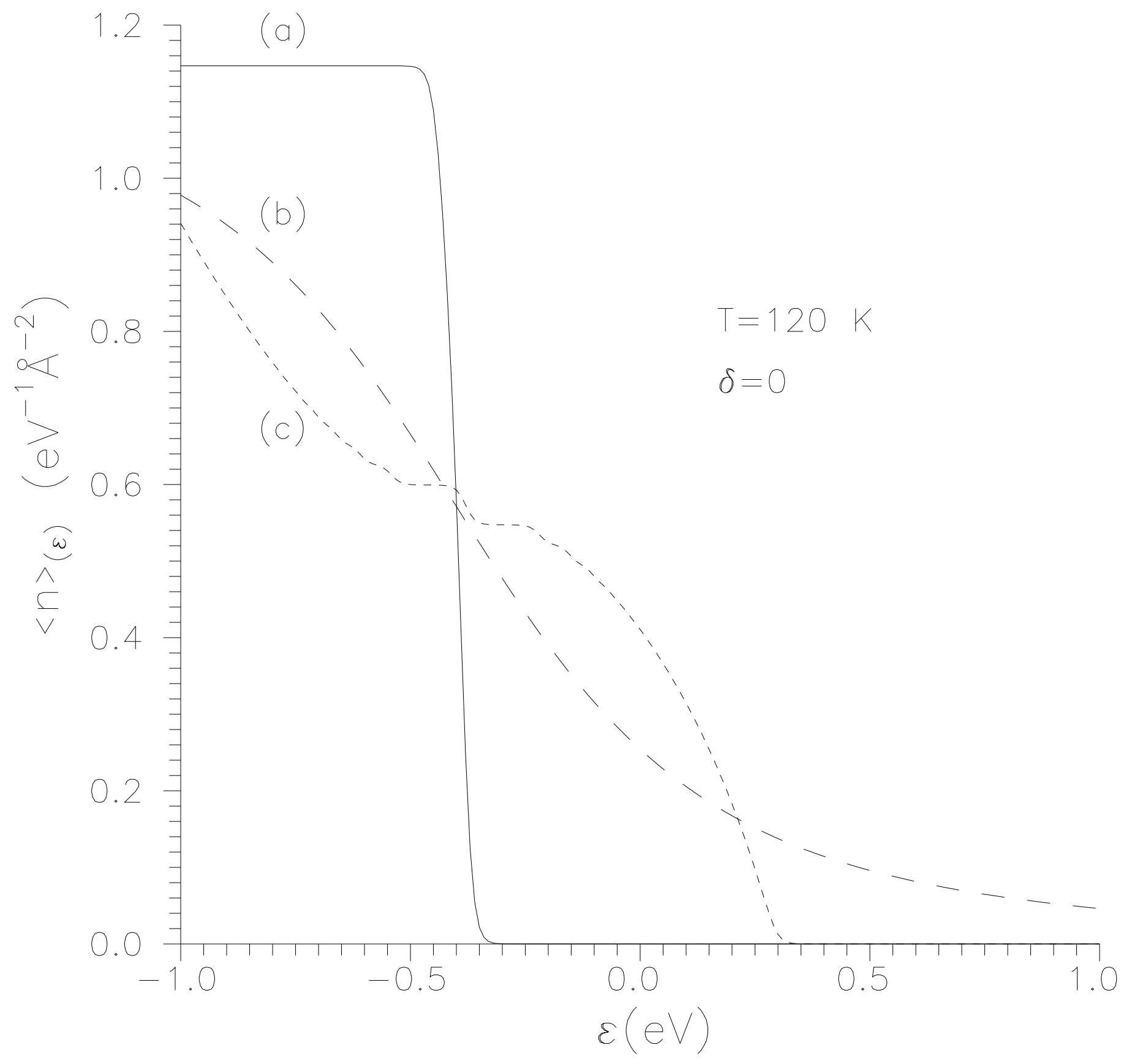


Fig. 2

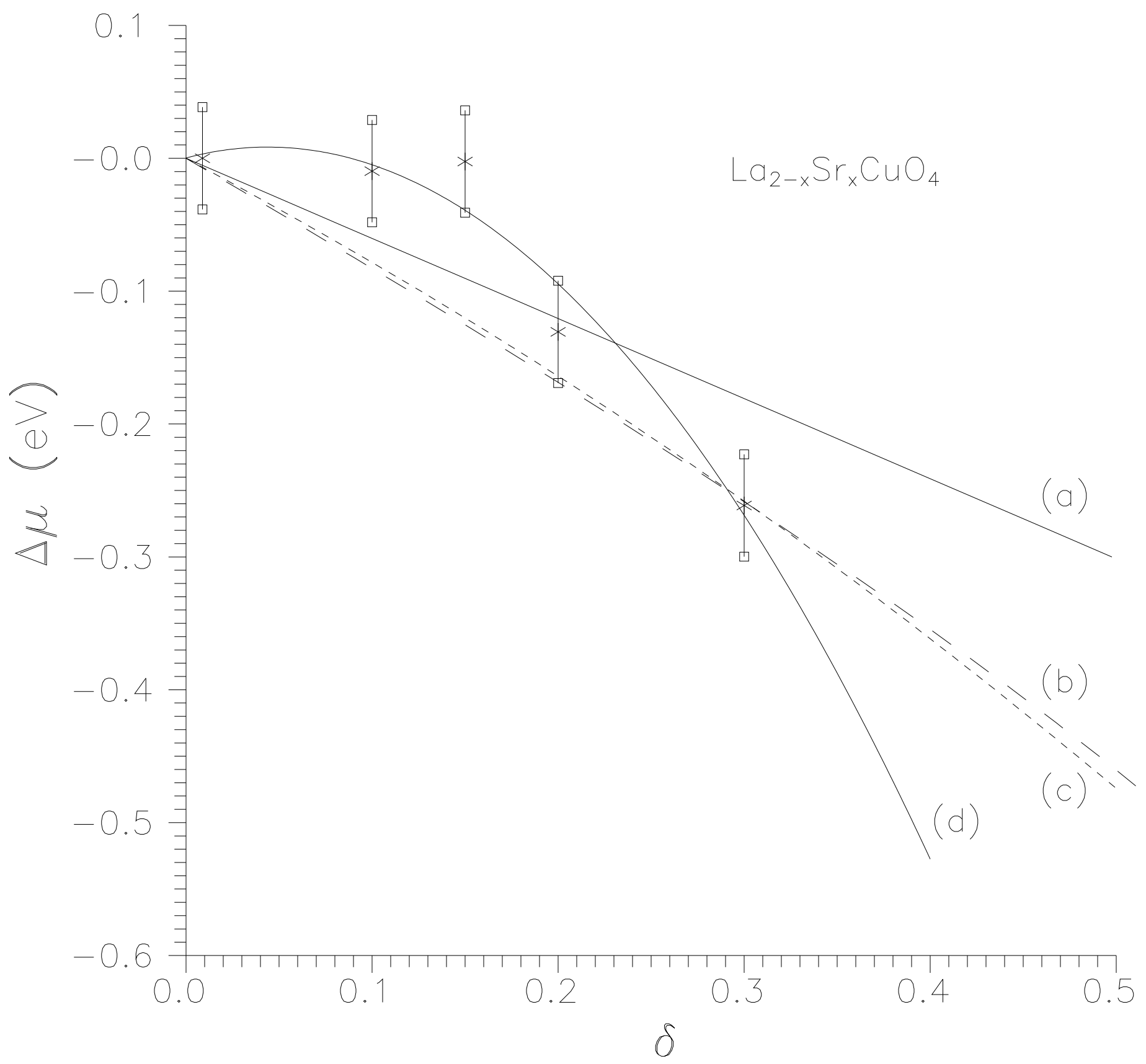

